\title{
Descriptive Analysis of Long- and Intermediate-Acting Insulin and Key Safety Outcomes in Adults with Type 2 Diabetes Mellitus
}

\author{
Daniel J. Kent, BSPharm, PharmD, CDE; Cheryl N. McMahill-Walraven, MSW, PhD; \\ Catherine A. Panozzo, PhD, MPH; Pamala A. Pawloski, PharmD, BCOP, FCCP; \\ Kevin Haynes, PharmD, MSCE; James Marshall, MPH; Jeffrey Brown, PhD, MA; \\ Bernadette Eichelberger, PharmD; and Catherine M. Lockhart, MS, PharmD, PhD
}

\begin{abstract}
BACKGROUND: As new biosimilar and follow-on insulins enter the market, more data are needed on safety, effectiveness, and patterns of use for these products to inform prescriber and patient decision-making regarding treatment. Additionally, data are needed regarding real-world patterns of use to inform future studies comparing the safety and effectiveness of biosimilars to already approved agents for diabetes treatment.
\end{abstract}

OBJECTIVE: To analyze the medication use patterns, adverse events, and availability of glycated hemoglobin (A1c) values for adult patients with type 2 diabetes mellitus (T2DM) who use long-acting insulin (LAI) or neutral protamine Hagedorn (NPH), an intermediate-acting insulin.

METHODS: We used the Biologics and Biosimilars Collective Intelligence Consortium's (BBCIC) distributed research network (DRN) for this descriptive analysis. The analysis time frame was January 1, 2011, to September 30,2015 , and included patients continuously insured for at least 183 days before the first date of a filled prescription for LAI or NPH insulin alone or with rapid- or short-acting insulin or sulfonylureas, whether newly starting insulin or switching to a different product. Insulin exposure episodes were the unit of analysis, and patients were classified in cohorts according to treatment. We followed patients until end of health plan enrollment or the end of the study period. We used occurrence of a study outcome, switch to another medication regimen, discontinuation of the current medication, or study end date to mark the end of an insulin episode. We describe demographics and availability of A1c values for analysis. Study outcomes included severe hypoglycemic events and major adverse cardiac events (MACE).

RESULTS: We identified 103,951 patients with T2DM from a database of 39.1 million patients with commercial or Medicare Advantage pharmacy and medical benefits, who contributed 279,533 unique insulin exposure episodes. Most episodes (89\%) included patients using LAI, and $52 \%$ of patients contributed data to 2 or more exposure cohorts. Insulin episodes lasted an average of 3.5 months, and patients had an average follow-up of 8.6 months. The unadjusted rate of severe hypoglycemic events requiring medical attention was 96.9 per 10,000 patient-years at risk (10kPYR). The unadjusted incident MACE rate was 676.9 events per 10kPYR. 38,330 T2DM patients in the BBCIC DRN had a baseline A1c available, and of those, less than $50 \%$ had a follow-up A1c result.

CONCLUSIONS: Among patients with T2DM, our observed insulin patterns of use and rates of severe hypoglycemic outcomes and MACE are consistent with other studies. We noted a paucity of A1c results available, which implies that additional data sources may be needed to augment the BBCIC DRN.

J Manag Care Spec Pharm. 2019;25(11):1162-71

Copyright $\odot 2019$, Academy of Managed Care Pharmacy. All rights reserved.

\section{What is already known about this subject}

Patients with type 2 diabetes mellitus (T2DM) may use multiple medications, and characterizing patterns of use is often difficult because of this heterogeneity.

Patients with T2DM benefit from using insulin, and such patients may adopt follow-on insulins in the future.

Indentification of potential data sources to monitor insulin use and patient outcomes is needed to better understand future uptake and patient outcomes when using follow-on insulins.

\section{What this study adds}

With the Biologics and Biosimilars Collective Intelligence Consortium (BBCIC) distributed research network (DRN), we described insulin exposures and insulin-related outcomes for patients with T2DM in a large, commercially insured population of patients.

The insulin use, baseline covariates, and insulin-related outcomes we observed in the BBCIC DRN are similar to previous studies among patients with T2DM.

A n estimated 29 million people in the United States have type 2 diabetes mellitus (T2DM), representing $9 \%$ of the total population. ${ }^{1}$ Approximately 6 million patients with T2DM use insulin replacement to manage their diabetes. ${ }^{1}$ The patent expiration of long-acting insulin (LAI) or intermediateacting insulin products, such as neutral protamine Hagedorn (NPH) insulin, has increased therapeutic options for patients with diabetes. The U.S. Food and Drug Administration (FDA) approved Basaglar (insulin glargine injection) and Admelog (insulin lispro injection) in 2017-2018, and review of new biosimilar insulins and innovative insulin products is ongoing. ${ }^{2}$ The FDA's accelerated approval processes for biosimilars and follow-on biologics require only comparative safety and efficacy data to the originator product, reducing lengthy clinical trial time frames. Patients and providers are interested in assurances regarding safety and effectiveness of biosimilar products when used in clinical practice..$^{3-6}$ Real-world evidence is one potential source of information that addresses these concerns. There is a paucity of research regarding the real-world comparative 
safety and effectiveness of biosimilars and follow-on biologics and their reference biologics, including insulins. In an initial effort to begin to fill this research gap, we have conducted a descriptive analysis to evaluate the fitness and robustness of a potential data source that can be used for future comparative analyses of biosimilars.

For this research, we used the Biologics and Biosimilars Collective Intelligence Consortium (BBCIC) distributed research network (DRN) to determine data availability, describe potential study populations, and define rates of severe adverse events among patients with T2DM and insulin exposure. The $\mathrm{BBCIC}$ is a nonprofit public service initiative formed to provide scientific evidence on the comparative safety and effectiveness of biosimilars and innovator biologics. The BBCIC DRN is composed of commercial and Medicare Advantage health insurance providers and leverages the FDA Sentinel data structure and analytical tools for its research purposes. ${ }^{7,8}$ More information on the BBCIC is available at www.bbcic.org.

In this study, we identified and described the patient cohorts of interest, described patterns of medication use, and evaluated outcomes among adult patients (aged 18 years and over) with T2DM. We focused on LAI and NPH insulin products to give a comprehensive description of background insulin use in this population and described the potential users of biosimilars and follow-on insulins. We also reported patient outcomes by (a) use of oral second-generation sulfonylureas (SU) and rapid-acting insulin (R) in conjunction with LAI or NPH insulin; (b) the frequency of preexisting cardiovascular, renal, neurologic, and retinal conditions; and (c) the percentage of patients with a history of hypoglycemic events. We then evaluated the outcomes of serious hypoglycemic events, major adverse cardiac events (MACE), and availability of hemoglobin Alc (Alc) values.

\section{Methods}

The BBCIC DRN leverages the FDA Sentinel Common Data Model (SCDM; V6 used in this analysis) and includes commercial and Medicare Advantage administrative claims data from 2 national health insurers (Aetna and Healthcore [Anthem]) and 3 regional health insurers (Kaiser Permanente of Washington, Harvard Pilgrim Health Care, and HealthPartners), ${ }^{9,10}$ Each health plan participates in the FDA Sentinel initiative. ${ }^{7,8}$

\section{Study Design and Population}

We used the BBCIC DRN for this descriptive cohort study. The study period was January 1, 2011, to September 30, 2015, which included a baseline lookback period of 183 days before an insulin exposure episode. The lookback was to ensure that no outcomes of interest occurred during that period that could be attributed to a cause other than insulin exposure, thus establishing the incidence of an outcome. This is the typical baseline period used by the FDA Sentinel initiative analyses. ${ }^{11}$
Patients could contribute multiple exposure episodes to this study, provided they met all inclusion and exclusion criteria, including 6 months with no occurrence of outcomes of interest. Both new (e.g., incident) and current (e.g., prevalent) users of insulin were included, but the lookback period allowed for identification of new (incident) primary outcomes.

The study population included adults aged 18 years or over with commercial or Medicare Advantage medical and pharmacy insurance, a diagnosis of T2DM, and a dispensed LAI or NPH insulin product within the study period. We defined T2DM as at least 1 medical claim with a T2DM (250.x0 or 250.x2) International Classification of Diseases, Ninth Revision, Clinical Modification (ICD-9-CM) diagnosis code observed at any time in the claims history.

Similar to exclusion criteria often used in clinical trials, ${ }^{12-14}$ we used the modified Klompas algorithm to exclude patients with health insurance claims for T1DM and T2DM diagnoses, as well as claims for insulin pumps or related supplies, gestational diabetes, liver disease, dialysis, end-stage renal disease, amputations, hemoglobinopathy, hemolytic anemia, sickle cell anemia, and blood transfusion. We excluded any patients with an emergency department (ED) visit for hypoglycemia or an ED visit or hospitalization for a cardiovascular event in their claims 183 days before the index insulin episode date. We also truncated exposure episodes if any insulins except those listed below were dispensed, unless such insulins were used in combination with LAI or NPH insulin. Patients could switch between LAI or NPH insulin cohorts. All codes used in this analysis can be found at www.bbcic.org.

We used the following medication subgroups to categorize patients based on insulin exposure:

1. LAI: Long-acting insulin, including insulin detemir and glargine.

2. LAI + R: Long-acting insulin plus rapid- or short-acting insulin, including detemir or glargine plus regular, lispro, glulisine, or aspart insulin and combinations such as aspart protamine human/aspart, as well as aspart protamine human/aspart plus regular pork, beef, or human or lispro/ lispro-protamine combinations.

3. LAI +SU: Long-acting insulin plus second-generation SU agent, including insulin detemir and glargine with glimepiride, glipizide, glipizide/metformin, glyburide, or glyburide/metformin.

4. NPH: Intermediate-acting insulin, including Humulin, Novolin, isophane pork, beef, or human.

5. NPH+R: NPH insulin plus rapid- or short-acting insulin, including Humulin, Novolin, mixed isophane pork, beef, or human and regular as well as isophane pork, beef, or human plus regular pork, beef, or human.

6. NPH+SU: NPH insulin plus second-generation SU agent, including Humulin, Novolin, isophane pork, beef, or 
Episode 1

Episode 2

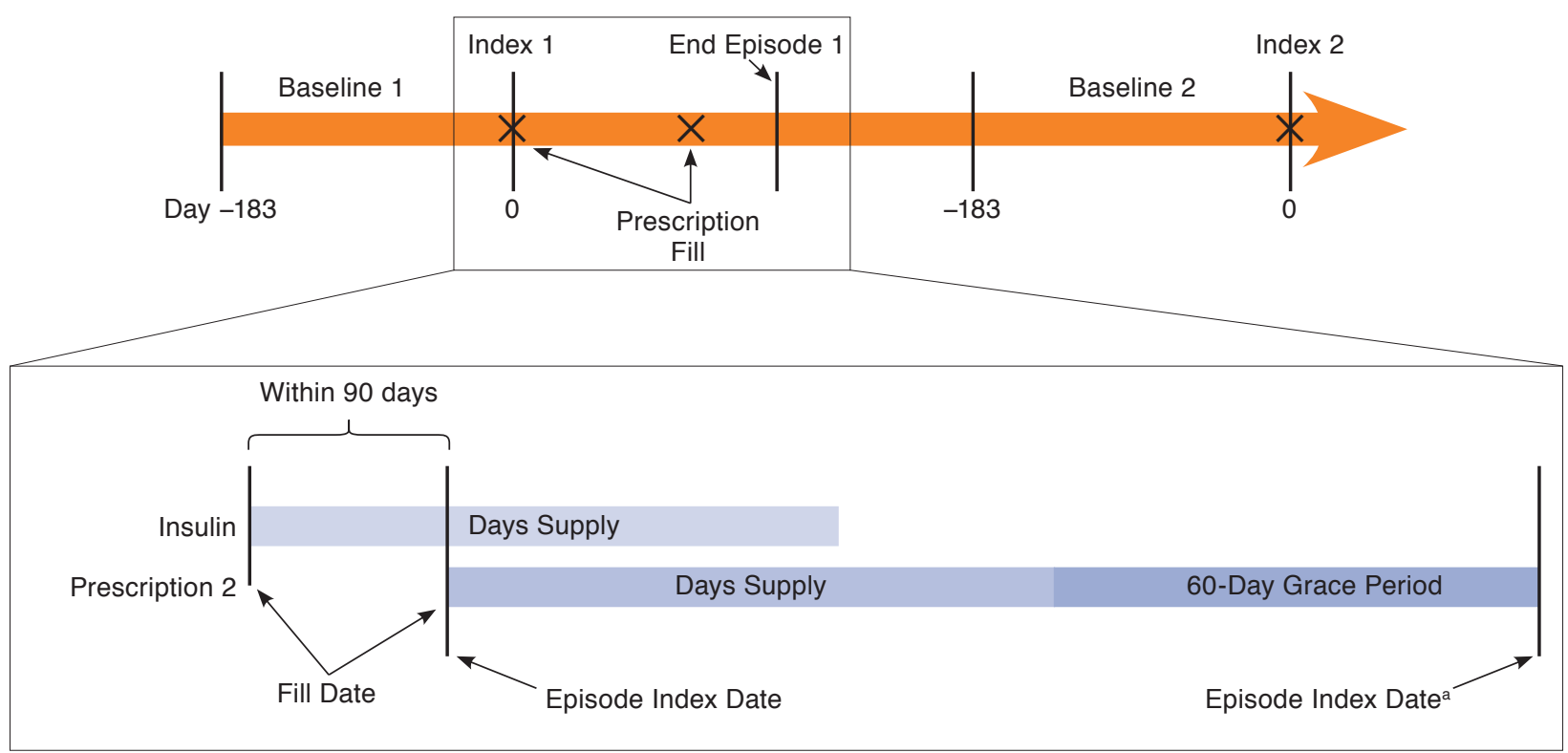

aAn epsiode can end due to the timeline illustrated here or earlier if a study outcome or other truncation event occurs.

human with glimepiride, glipizide, glipizide/metformin, glyburide, or glyburide/metformin.

Exposure to metformin was allowed within each of the 6 groups; however, an episode was truncated if a patient filled a prescription for oral antidiabetes agents other than those included in the exposure cohorts, including first-generation SUs (e.g., chlorpropamide, tolazamide); thiazolidinediones (TZD; e.g., pioglitazone, rosiglitazone); dipeptidyl peptidase-4 inhibitors (DPP-4; e.g., sitagliptin, saxagliptin); glucagon-like peptide-1 agonists (GLP-1; e.g., exenatide, liraglutide); and sodium glucose cotransporter 2 inhibitors (SGLT2; e.g., empagliflozin, canagliflozin).

\section{Unit of Analysis and Medication Exposure}

Insulin exposure episode was the unit of analysis for primary outcomes, with the insulin dispensing date as the index date of an exposure episode. An exposure episode began on the date of prescription fill for LAI or NPH and extended for the prescribed insulin days supply with a grace period of 60 days added to the end to account for imperfect adherence and changes to insulin dosage. Continuous medical and prescription drug insurance coverage was required, with a 45-day enrollment gap permitted. We analyzed the LAI and NPH cohorts with "+R" (long-acting plus rapid-acting insulin) or "+SU" (long-acting insulin plus oral SU) to describe patterns of care and characteristics of patients who may use LAI biosimilars, and outcomes for patients who are at increased risk of hypoglycemia and cardiovascular events. ${ }^{15-18}$ To be categorized in a combination cohort (e.g., LAI+R) for an episode, patients could fill these concomitant medications on the same day as LAI or NPH, or within 90 days following the LAI or NPH fill date. When a second agent was filled within this window, the episode was categorized in the combination cohort. For these groups, the dispensing date of the second agent (R or SU) defined the episode index date.

Exposure episode length was determined by days supplied reported from the pharmacy claim with a 60-day grace period to account for adherence and dosing variability. Following the FDA Sentinel analytic standard, an episode began at the index date and continued as long as there was no gap in insulin or other medication exposure of 60 days or greater observed. We ended the exposure episode if a patient's claims reflected 61 days or longer without a filled prescription for any of the medications. The next prescription filled for the patient began a new medication episode.

Time at risk was defined as the sum of all episode time. Patients stopped contributing time at risk at the point when an episode was either truncated or ended but could resume contributing time at risk at the start of the next episode. 


\section{FIGURE 2 CONSORT Diagram for Study Cohort Selection}

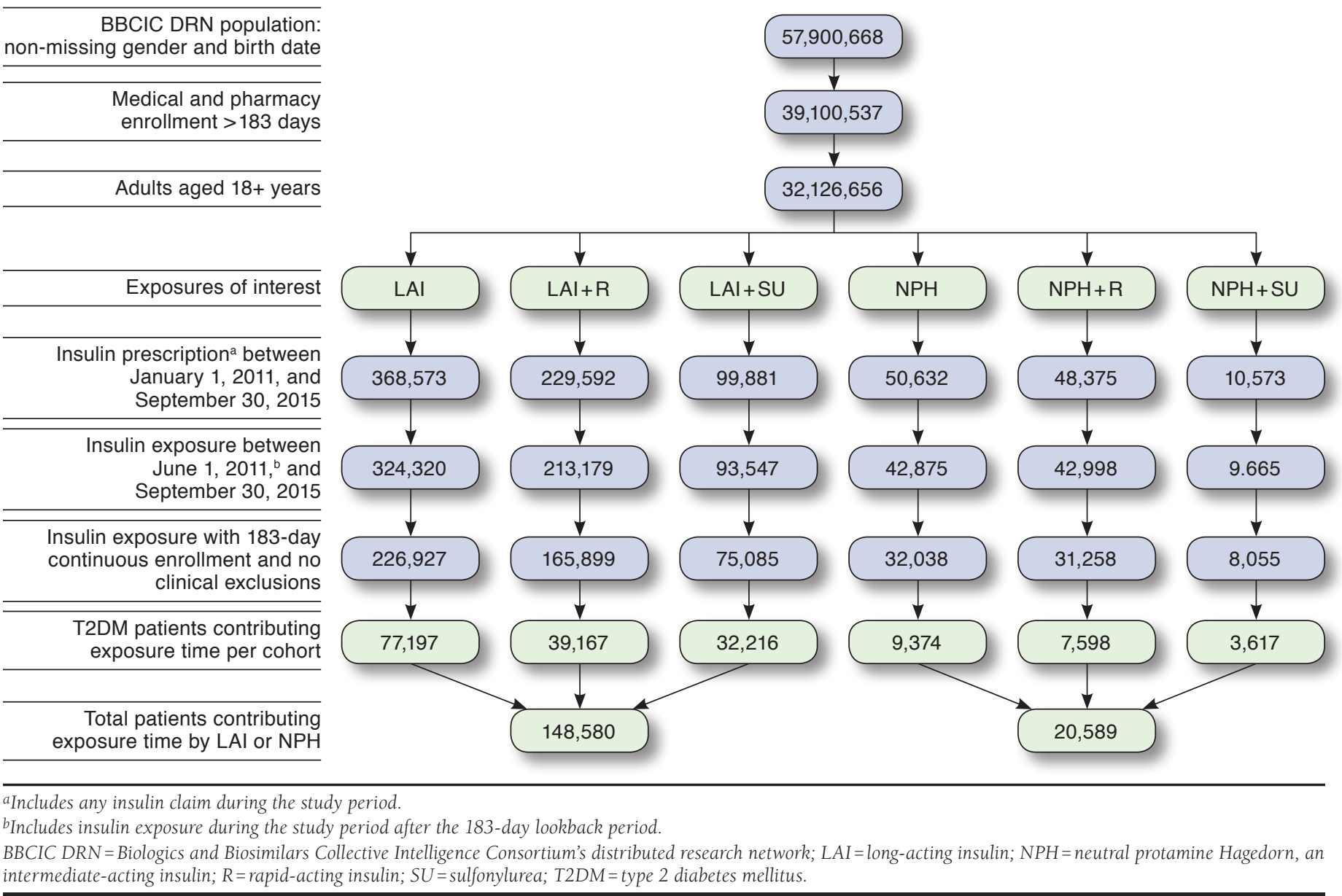

Patients could contribute more than 1 episode with different index dates if they met all inclusion criteria at the time of each new exposure; those who switched medications during the study period were assigned to the cohort for the new medication. Patients were further stratified into those who contributed to 1 medication cohort versus those in multiple cohort types (i.e., LAI/NPH only vs. LAI/NPH as well as LAI/NPH+SU).

We truncated a medication exposure episode for the following reasons: subject loss to follow-up (e.g., end of insurance enrollment), medication regimen change during an exposure, study outcome attainment, or end of study observation period. Medication regimen changes that resulted in truncation include (a) use of an insulin in a category different from the initial exposure insulin; (b) use of oral antidiabetes medications including first-generation SU agents; or (c) use of DPP-4, GLP-1, SGLT-2, or TZD medications alone or in combination with insulin. After a truncated insulin exposure episode, the patient could have another episode with a new 183-day lookback baseline period (Figure 1).

\section{Baseline Characteristics}

We described the following clinical and demographic characteristics for patients: age (18-49, 50-64, 65-79, and 80+ years); gender; insulin type; health service utilization (e.g., number of hospitalizations, ED, and ambulatory visits); medication measures (i.e., use of metformin and other antidiabetes medications); and specific comorbidities incorporated into a combined comorbidity index (includes conditions found in the Romano adoption of the Charlson Comorbidity Index and the Elixhauser Comorbidity Index, including complicated diabetes, cardiac, renal, or neurological comorbidities). ${ }^{19,20}$ We accounted for level of illness by combined comorbidity index along with ED use, inpatient hospitalization, and metformin use in the 183 days before each insulin episode in the study period. ${ }^{21}$ 
TABLE 1 Baseline Characteristics, Insulin Episodes, and Dispensing Patterns Among All Patients with a Recorded Use of LAI or NPH Insulin

\begin{tabular}{|c|c|c|c|c|c|c|}
\hline \multicolumn{7}{|c|}{ Insulin Episodes } \\
\hline Unique patients with at least 1 episode & \multicolumn{6}{|c|}{$\mathrm{N}=103,951$} \\
\hline \multirow{2}{*}{ Total number of episodes } & \multicolumn{6}{|c|}{$\mathrm{N}=279,533$} \\
\hline & LAI & $\mathrm{LAI}+\mathrm{R}$ & $\mathrm{LAI}+\mathrm{SU}$ & NPH & $\mathrm{NPH}+\mathrm{R}$ & $\mathrm{NPH}+\mathrm{SU}$ \\
\hline Insulin episodes, $\mathrm{n}$ & 128,686 & 68,074 & 50,682 & 14,540 & 12,197 & 5,354 \\
\hline Episodes per patient, mean (SD) & $1.67 \quad(1.2)$ & $1.74 \quad(1.3)$ & $1.57 \quad(1.1)$ & $1.55 \quad(1.2)$ & $1.61 \quad(1.2)$ & $1.48 \quad(1.0)$ \\
\hline Months per episode, mean (SD) & $2.28 \quad(4.3)$ & $3.84 \quad(5.3)$ & $(5.3)$ & $4.56 \quad(8.4)$ & $4.92 \quad(6.6)$ & $6.96 \quad(9.5)$ \\
\hline Months of follow-up, ${ }^{\text {a }}$ mean (SD) & $3.72 \quad(6.6)$ & $6.72 \quad(8.8)$ & $(7.9)$ & $6.96(11.9)$ & $7.92(10.4)$ & $10.32(13.2)$ \\
\hline Dispensing per patient, mean (SD) & $2.91 \quad(4.0)$ & $3.79 \quad(5.1)$ & $3.10 \quad(4.0)$ & $4.00 \quad(5.9)$ & $4.33 \quad(6.1)$ & $3.99 \quad(5.0)$ \\
\hline Dispensing per episode, mean (SD) & $1.75 \quad(2.8)$ & $2.18 \quad(3.4)$ & $1.97 \quad(2.7)$ & $2.58 \quad(4.5)$ & $2.70 \quad(4.4)$ & $2.69 \quad(3.6)$ \\
\hline Days supplied per patient, mean (SD) & $75.8(142.8)$ & $116.4(180.1)$ & $98.5(170.3)$ & $157.9(290.1)$ & $146.8(225.8)$ & $217.3(323.1)$ \\
\hline Days supplied per episode, mean (SD) & $45.5(102.4)$ & $67.0(122.2)$ & $62.6(125.4)$ & $101.8(220.2)$ & $91.5(160.8)$ & $146.8(250.5)$ \\
\hline \multicolumn{7}{|c|}{ Patient Characteristics by Insulin Episode } \\
\hline Female, ${ }^{\mathrm{b}} \mathrm{n}(\%)$ & $59,201 \quad(46)$ & $32,004 \quad(47)$ & $22,735 \quad(45)$ & 7,586 & 6,314 & 2,628 \\
\hline \multicolumn{7}{|l|}{ Age groups in years, ${ }^{\mathrm{c}} \mathrm{n}(\%)$} \\
\hline $18-49$ & 39,004 & $19,202 \quad(28)$ & 12,232 & 3,983 & 3,307 & 1,099 \\
\hline $50-64$ & $69,004 \quad(54)$ & $37,462 \quad(55)$ & 27,872 & 6,739 & 5,722 & 2,523 \\
\hline $65-79$ & $17,603 \quad(14)$ & $9,790 \quad(14)$ & $9,130 \quad(18)$ & $3,154 \quad(22)$ & 2,583 & 1,441 \\
\hline $80+$ & $3,075 \quad(2)$ & $1,620 \quad(2)$ & 1,448 & 664 & 585 & 291 \\
\hline \multicolumn{7}{|c|}{ Clinical Characteristics } \\
\hline & \multicolumn{3}{|c|}{ Any LAI } & \multicolumn{3}{|c|}{ Any NPH } \\
\hline Mean age, years (SD) & \multicolumn{3}{|c|}{$55.1(11.5)$} & \multicolumn{3}{|c|}{$57.1(12.6)$} \\
\hline Combined comorbidity index, mean (SD) & \multicolumn{3}{|c|}{$0.2 \quad(1.3)$} & \multicolumn{3}{|c|}{$0.5 \quad(1.4)$} \\
\hline Gastroparesis, \% & \multicolumn{3}{|c|}{0.3} & & 0.3 & \\
\hline Hyperlipidemia, \% & & 41.9 & & & 31.7 & \\
\hline Hypertension, \% & & 53.7 & & & 47.6 & \\
\hline Nephropathy and CKD, \% & & 4.1 & & & 8.1 & \\
\hline Obesity and abnormal weight gain, $\%$ & & 11.8 & & & 13.6 & \\
\hline Peripheral neuropathy, \% & & 6.4 & & & 13.5 & \\
\hline Retinopathy, \% & & 7.2 & & & 11.0 & \\
\hline & & ealth Service U & ization & & & \\
\hline Ambulatory visits, mean (SD) & & $5.2(6.2)$ & & & $5.1 \quad(6.3)$ & \\
\hline Emergency department visits, mean (SD) & & $0.2 \quad(0.5)$ & & & $0.1 \quad(0.5)$ & \\
\hline Inpatient hospital stays, mean (SD) & & $0.1 \quad(0.3)$ & & & $0.1 \quad(0.3)$ & \\
\hline Other ambulatory visits, mean (SD) & & $1.7 \quad(4.5)$ & & & $4.4 \quad(6.0)$ & \\
\hline Prescriptions dispensed, mean (SD) & & $16.1(14.3)$ & & & $15.4(13.3)$ & \\
\hline Generic prescriptions dispensed, mean (SD) & & $6.5 \quad(4.3)$ & & & $7.0 \quad(4.4)$ & \\
\hline Unique drug classes, mean (SD) & & $6.1 \quad(4.1)$ & & & $6.7 \quad(4.2)$ & \\
\hline Metformin use, $\%$ & & 46.8 & & & 50.7 & \\
\hline
\end{tabular}

Note: Results are based on episodes, and each patient may contribute multiple episodes across insulin exposures.

a Follow-up is defined as time from index insulin dispensing date to end of insulin episode. End of episode can be because of insurance disenrollment, death, end of data, or end of dispensed days of insulin plus 60 days extension.

${ }^{b}$ Number of episodes observed for female patients contributing time to the exposure category.

'Number of episodes observed for patients in each age category contributing time to the exposure category.

$C K D=$ chronic kidney disease; $L A I=$ long-acting insulin; $N P H=$ neutral protamine Hagedorn $(N P H)$, an intermediate-acting insulin; $R=$ rapid-acting insulin; $S D=$ standard deviation; $S U=$ sulfonylurea.

\section{Primary Outcomes}

Because addition of regular insulin and an SU has been shown to increase hypoglycemic and cardiovascular risk, primary outcomes were stratified by 3 combined insulin cohorts: (a) LAI and NPH, (b) $\mathrm{LAI}+\mathrm{R}$ and $\mathrm{NPH}+\mathrm{R}$, and (c) $\mathrm{LAI}+\mathrm{SU}$ and $\mathrm{NPH}+\mathrm{SU} \cdot{ }^{15-18}$ Hypoglycemic and MACE outcomes were identified using diagnosis codes and procedure codes. ${ }^{22}$ We included the first qualifying hypoglycemic event and the first qualifying MACE following the index insulin dispensing for each patient. In other words, among the 
prevalent (new and existing) users of insulin, we identified incident (new) events. For patients with multiple events, we classified an event by the exposure in the episode when the event occurred.

Hypoglycemia was defined using the following ICD-9-CM codes with the modified Ginde algorithm: (a) an ED visit with at least 1 diagnosis code of 250.0, 250.1, 250.2, 270.3, 775.0, or 775.6; or (b) ED visit with a diagnosis code of 250.8x, without the following codes: 251.0, 251.1, 251.2, 270.3, 775.0, 775.6, 259.8, 272.7, 681.xx, 682.xx, 686.9x, 707.x, 709.3, 730.0730.2 , or $731.8 .^{23} \mathrm{MACE}$ is a composite endpoint of hospitalization or ED visit for stroke, acute myocardial infarction (AMI) or unstable angina (including occlusion without infarction or coronary insufficiency), cardiac or carotid revascularization procedure, or other heart disease (including heart failure) defined using a combination of ICD-9-CM and Healthcare Common Procedural Coding System codes. ${ }^{24-26}$

\section{Secondary Outcomes}

We measured the availability of Alc values and created 3 categories: no Alc results available, any baseline Alc (i.e., within 183 days of index date), and any study period Alc (baseline or exposure period). Of the 5 data partners participating in this study, 3 contributed laboratory values to the BBCIC DRN. FDA Sentinel tools classify laboratory values in categories, so our analysis was limited to observing counts rather than analyzing Alc as a continuous variable. Alc values were categorized as Alc less than or equal to 6.5, between 6.5 and 7.0, between 7.0 and 8.0, and greater than 8.0.

\section{Statistical Methods}

We described continuous measures with means and standard deviations and categorical measures with counts, frequencies, and percentages. This study used a distributed analytic approach in which each site maintained data locally. Only aggregate data, and no individual patient-level data, were shared outside the study sites. Measures that involved patientlevel data, such as the calculation of means and standard deviations, were performed by ad hoc programs with only final aggregated results provided to $\mathrm{BBCIC}$. The BBCIC Coordinating Center at the Harvard Pilgrim Health Care Institute (HPHCI) used publicly available Sentinel analytic tools to create analytic code that was securely sent to each local site to create the study cohort. The HPHCI Institutional Review Board (IRB) and each data partner site IRB reviewed the protocol and determined it did not meet the definition for human subject research.

\section{Results}

Over 39.1 million patients met the study inclusion criteria of at least 183 days of medical and drug coverage during the study period; 82\% (32.1 million) were adults aged 18 years or over (Figure 2). Within each of these exposure cohorts,

\begin{tabular}{|c|c|c|}
\hline \multicolumn{3}{|c|}{$\begin{array}{l}\text { Insulin Episodes and Dispensing } \\
\text { Patterns Among All Patients with } \\
\text { Recorded Use of LAI or NPH Insulin } \\
\text { Contributing Either } 1 \text { Insulin Episode } \\
\text { Only or } 2 \text { or More Insulin Episodes }\end{array}$} \\
\hline \multicolumn{3}{|c|}{ Insulin Episodes } \\
\hline Unique patients with at least 1 episode & \multicolumn{2}{|c|}{$\mathrm{N}=103,951$} \\
\hline \multirow[t]{2}{*}{ Total number of episodes ${ }^{a}$} & \multicolumn{2}{|c|}{$\mathrm{N}=279,337$} \\
\hline & $\begin{array}{l}\text { Any Exposure, } \\
1 \text { Cohort Only }\end{array}$ & $\begin{array}{c}\text { Any Exposure, } \\
2 \text { or More } \\
\text { Cohorts }\end{array}$ \\
\hline Patients contributing to episodes, $n$ & 49,704 & 54,247 \\
\hline Insulin episodes, $\mathrm{n}$ & 77,538 & 201,799 \\
\hline Episodes per patient, mean (SD) & $1.56 \quad(1.2)$ & $3.72 \quad(2.5)$ \\
\hline Months per episode, mean (SD) & $(6.7)$ & $2.64 \quad(4.7)$ \\
\hline Months of follow-up, ${ }^{a}$ mean (SD) & $(9.7)$ & $10.08 \quad(11.2)$ \\
\hline Dispensing per patient, mean (SD) & $(5.8)$ & $6.53 \quad(6.1)$ \\
\hline Dispensing per episode, mean (SD) & $2.62 \quad(4.3)$ & $1.75 \quad(2.5)$ \\
\hline Days supplied per patient, mean (SD) & $134.81(219.4)$ & $189.32(239.0)$ \\
\hline Days supplied per episode, mean (SD) & $86.41(165.5)$ & $50.85(109.8)$ \\
\hline \multicolumn{3}{|c|}{$\begin{array}{l}\text { aThe total number of exposures observed is slightly less than those defined by insulir } \\
\text { cohort because of rounding when calculating the combined cohorts. } \\
\text { bIncludes all exposure cohorts: } L A I, L A I+R, L A I+S U, N P H, N P H+R \text {, and } \\
N P H+S U \text {. } \\
\text { LAI=long-acting insulin; NPH =neutral protamine Hagedorn }(N P H) \text {, an } \\
\text { intermediate-acting insulin; } R=\text { rapid-acting insulin; } S D=\text { standard deviation; } \\
\text { SU = sulfonylurea. }\end{array}$} \\
\hline
\end{tabular}

$55 \%-76 \%$ were excluded because of a diagnosis of T1DM in claims, clinical exclusions (e.g., history of myocardial infarction), or observation of an outcome in the baseline period. These exclusions yielded final counts of 77,197 patients contributing to the LAI cohort, 39,167 patients contributing to the LAI+R cohort, 32,216 patients contributing to the LAI+SU cohort, 9,374 patients contributing to the NPH cohort, 7,598 patients contributing to the NPH+R cohort, and 3,617 patients contributing to the $\mathrm{NPH}+\mathrm{SU}$ cohort.

A total of 103,951 patients contributed at least 1 insulin exposure episode and met study criteria for inclusion. A total of 279,533 insulin episodes were included in this analysis. Table 1 shows insulin exposures, dispensings, and patient characteristics for each insulin cohort. There were 128,686 episodes in the LAI cohort, 68,074 episodes in the LAI+R cohort, 50,682 episodes in the LAI+SU cohort, 14,540 episodes in the NPH cohort, 12,197 episodes in the NPH+R cohort, and 5,354 episodes in the NPH+SU cohort during the study period. Patients could be included in more than 1 group because of changing treatment regimens over time. Overall, insulin episodes lasted an average of 3.5 months, and patients had an average follow-up of 8.6 months. The average episode duration ranged from 2.3 to 3.8 months in the LAI cohorts and from 4.6 to 7.0 months in the NPH cohorts. Follow-up time in the LAI cohorts ranged from 3.7 to 6.7 months and 7.0 to 10.3 months in the NPH cohorts. 


\section{TABLE 3 Outcomes Among Patients with T2DM by Type of Insulin Used}

\begin{tabular}{|c|c|c|c|c|}
\hline \multicolumn{5}{|c|}{ Hypoglycemia } \\
\hline \multirow{2}{*}{ Unique patients evaluated for outcomes } & \multicolumn{4}{|c|}{$\mathrm{N}=102,960^{\mathrm{a}}$} \\
\hline & LAI or NPH & $(\mathrm{LAI}$ or $\mathrm{NPH})+\mathrm{R}$ & $(\mathrm{LAI}$ or $\mathrm{NPH})+\mathrm{SU}$ & Any Exposure ${ }^{b}$ \\
\hline Insulin episodes & 139,756 & 77,870 & 55,278 & 272,904 \\
\hline Episodes with events & 304 & 309 & 156 & 769 \\
\hline Patients with events & 302 & 306 & 156 & 721 \\
\hline Patients with events per 10kPYR & 102.6 & 111.5 & 88.8 & 96.9 \\
\hline Episodes per patient with events, mean (SD) & $1.94(1.2)$ & $2.08(1.5)$ & $1.89(1.3)$ & $2.10(1.5)$ \\
\hline Episodes with events per 10kPYR & 103.3 & 112.6 & 88.9 & 103.4 \\
\hline \multicolumn{5}{|c|}{ MACE } \\
\hline Episodes with events & 2,061 & 2,234 & 1,231 & 5,526 \\
\hline Patients with events & 2,020 & 2,166 & 1,205 & 5,036 \\
\hline Patients with events per 10kPYR & 702.1 & 811.5 & 703.5 & 676.9 \\
\hline Episodes per patient with events, mean (SD) & $1.83(1.4)$ & $1.94(1.4)$ & $1.73(1.2)$ & $1.98(1.5)$ \\
\hline Episodes with events per 10kPYR & 716.3 & 837.0 & 718.7 & 742.8 \\
\hline
\end{tabular}

\section{Insulin Episode and Patient Characteristics}

Insulin episode and patient characteristics are shown in Table 1. A total of $46 \%$ of episodes in the LAI cohort occurred in females, compared with $52 \%$ in the NPH cohort. In the LAI+R and $\mathrm{NPH}+\mathrm{R}$ cohorts, $47 \%$ and $52 \%$ of episodes occurred in females, respectively, and in the LAI+SU and NPH+SU cohorts $45 \%$ and $49 \%$ of episodes occurred in females, respectively. The majority of episodes occurred in patients aged 50 to 64 years. Approximately half $(47 \%)$ of LAI episodes included a metformin dispensing in the previous 183 days, while $51 \%$ of NPH episodes included a metformin dispensing.

During baseline, 54\% of LAI episodes had evidence of hypertension; $42 \%$ had evidence of hyperlipidemia; $12 \%$ had evidence of obesity or abnormal weight gain; and 6\% had evidence of peripheral neuropathy. In the NPH episodes, we saw evidence of hypertension in $48 \%$ of episodes, hyperlipidemia in $32 \%$, obesity or abnormal weight gain in $14 \%$, and peripheral neuropathy in 14\% of episodes. Patients with LAI episodes had an average of 5 ambulatory visits and 16 prescriptions filled in 6 drug classes during the study period, while those with NPH episodes had an average of 5 ambulatory visits and 15 prescriptions filled in 7 drug classes in the 183 days before index insulin dispensing.

Of the 103,951 T2DM patients, $48 \%$ were categorized in only 1 type of insulin exposure cohort, accounting for $28 \%$ of insulin episodes (Table 2). The remaining 52\% were categorized in multiple exposure cohorts (e.g., NPH followed by $\mathrm{NPH}+\mathrm{R}$ ), accounting for $72 \%$ of insulin episodes. Patients with a single episode type had 1.6 episodes per patient, an average of 4.1 insulin prescriptions dispensed, and an average of
135 days supply. As compared with patients with a single episode type, patients with multiple insulin exposure types had more episodes (3.7) and insulin prescriptions (6.5) dispensed per patient, and an average of 54 more days of insulin exposure (189 days).

\section{Primary Outcomes}

Table 3 displays the 2 primary outcomes by insulin episode group (LAI vs. NPH). The unadjusted hypoglycemic event rates for all patients with T2DM was 96.9 events per 10,000 person-years at risk (10kPYR), ranging from 88.8 for (LAI or $\mathrm{NPH})+\mathrm{SU}$ patients to 111.5 for patients using (LAI or NPH) $+\mathrm{R}$. The unadjusted incident MACE rates for patients with T2DM was 676.9 per 10kPYR, ranging from 702.1 for LAI or NPH patients to 811.5 for patients using (LAI or NPH) + R.

\section{Secondary Outcomes}

A total of 38,330 patients in the BBCIC DRN had a baseline Alc value; of those, 18,507 (48\%) had both baseline and study period Alc values. For those patients with a baseline Alc value, the first study period Alc after the insulin dispensing index date occurred within 2.9 months, compared with 4.2 months for patients without a baseline Alc value.

\section{Discussion}

This descriptive analysis used the BBCIC DRN to describe the demographics and medication use among adult patients with T2DM. We also characterized the insulin-related outcomes of hypoglycemic events and MACE, plus availability of Alc levels. 
The baseline characteristics reported are consistent with those reported in clinical trials and observational studies. Average age for our population was comparable to those in other published observational and prospective cohort studies. ${ }^{27-31}$ Our population had a high percentage of patients with preexisting cardiovascular conditions. In order of prevalence, we observed hypertension, other heart disease (including heart failure), ischemic heart disease, nephropathy/chronic kidney disease, stroke/carotid revascularization, end-stage renal disease, AMI, and coronary revascularizations as seen in other observational and clinical trials. However, unlike other studies such as LEADER, we excluded patients with a history of cardiovascular events. Metformin use was consistent with longer term persistence studies. ${ }^{32}$ The use of metformin and SU in the LAI group was consistent with that reported in the literature but was almost 3 times higher than in the NPH cohort. ${ }^{33,34}$

Our results using unadjudicated events based on claims data were in the middle of published MACE rates. Our unadjusted MACE event rate of 676.9 per 10kPYR was higher than those seen in clinical trials of diabetes medications among prevalent diabetes, which varied from 278 to 528 events per 10kPYR, respectively. ${ }^{35-38}$ Our MACE composite criteria most closely resembled those of 2 trials, but overall we observed MACE outcomes generally lower than those observed in the LEADER and ORIGIN trials. The LEADER trial had T2DM MACE outcomes of 790 events per 10kPYR with transient ischemic attack, coronary revascularization, unstable angina, and congestive heart failure, which is higher than our MACE event rates except for our LAI/NPH+R group rate (837 events per 10kPYR). ${ }^{39}$ For the ORIGIN trial, when primary and secondary MACE rates were included ( 846 events per 10kPYR), the rates were also higher than our findings. ${ }^{40}$ In both the LEADER and ORIGIN trials, patients with previous cardiovascular disease were included in the study, whereas we excluded such patients from analysis, which likely explains our lower findings of MACE, since those with preexisting cardiovascular disease are also more predisposed to MACE.

As expected, the $\mathrm{LAI}+\mathrm{R} / \mathrm{NPH}+\mathrm{R}$ group experienced a higher rate of hypoglycemia events (111.5 events per 10kPYR) compared with the other studies of insulin users. ${ }^{41}$ Although direct comparisons to clinical trials are difficult given the cohorts and data available (e.g., claims documentation vs. clinical documentation), our unadjusted hypoglycemia rates were close to trial results and consistent with other claims studies. ${ }^{42}$

\section{Limitations}

We note a few limitations for this study. We observed a relatively short enrollment period for available patients. The average length of enrollment across the patients in the BBCIC DRN was 17 months, which included the 183-day lookback period, and was similar to the SCDM characterization. ${ }^{43}$ The short time frame was caused by the real-world phenomena of a commercially insured population, with employment changes or employers changing health insurance carriers influencing plan enrollment, as well as Medicare Advantage patients who may have changed enrollment plans or enrolled in traditional fee-for-service Medicare. ${ }^{44,45}$ The insulin exposure period for the population was 7 months for T2DM patients with 1 insulin episode and 10.8 for patients with T2DM and multiple insulin episodes. Our unadjusted cardiovascular outcome events may have reflected our shorter baseline period, study time length, and the average patient enrollment. ${ }^{46,47}$

Because of limitations of the FDA Sentinel tools, the results of this study were reported based on insulin episode rather than on a patient level. This can make interpretation of the results confusing and requires attention when reporting outcomes. Additionally, as these data cover the years 2011-2015, patterns of care for T2DM may have changed since that time, limiting the applicability of some of our findings.

The true treatment duration is difficult to discern from prescribed days supply with insulin use, compared with other chronic medications, because of variable dosing that is often observed to maintain blood glucose stability in patients with diabetes. We attempted to account for this variability, and the risk of lack of adherence, by including a 60-day grace period at the end of the dispensed days supply. It is likely that the true exposure period was either under- or overestimated; we cannot know which. This is an imperfect approach but reflects the inherent limitation in using claims data to evaluate drugs with variable dosing.

Finally, despite a 183-day minimum time frame, only 38,330 patients with T2DM had a baseline Alc. Only 3 of 5 research partners contributed laboratory data to this study, and the data were not complete for all patients with Alc records available. Laboratory measures are not always captured in claims, and without access to electronic medical records or other records of patient care, laboratory measures are often lacking at the level of insurance claims. Also, the FDA Sentinel tools classify laboratory values in categories, so our analysis was limited to observing counts rather than analyzing Alc as a continuous variable.

\section{Conclusions}

For this descriptive study, we used the BBCIC DRN to determine the availability of key data elements, describe relevant populations, and define rates of severe hypoglycemic events, MACE, and Alc result availability among patients with T2DM exposed to long- or intermediate-acting insulins. In this study, we found a large cohort of patients with T2DM and insulin exposure. This cohort could be used in future studies to evaluate the safety and effectiveness of biosimilars. 


\section{Authors}

DANIEL J. KENT, BSPharm, PharmD, CDE, Kaiser Permanente of Washington, Seattle; CHERYL N. MCMAHILL-WALRAVEN, MSW, PhD, Aetna, Blue Bell, Pennsylvania; PAMALA A. PAWLOSKI, PharmD, BCOP, FCCP, HealthPartners, Bloomington, Minnesota; and KEVIN HAYNES, PharmD, MSCE, HealthCore, Wilmington, Delaware. CATHERINE A. PANOZZO, PhD, MPH; JAMES MARSHALL, MPH; and JEFFREY BROWN, PhD, MA, Harvard Pilgrim Healthcare Institute, Boston, Massachusetts. BERNADETTE EICHELBERGER, PharmD, and CATHERINE M. LOCKHART, MS, PharmD, PhD, Biologics and Biosimilars Collective Intelligence Consortium, Alexandria, Virginia.

AUTHOR CORRESPONDENCE: Catherine M. Lockhart, MS, PharmD, PhD, Biologics and Biosimilars Collective Intelligence Consortium, 675 N. Washington St., Ste. 220, Alexandria, VA 22314. Tel.: 703.684.2646; E-mail: clockhart@bbcic.org.

\section{DISCLOSURES}

This study was coordinated and funded by the Biologics and Biosimilars Collective Intelligence Consortium (BBCIC) and represents the independent findings of the BBCIC Insulins Principal Investigator and the BBCIC Insulins Research Team. Lockhart is employed by the BBCIC and the Academy of Managed Care Pharmacy (AMCP). Eichelberger was employed by the BBCIC and AMCP at the time of this study. McMahill-Walraven is employed by Aetna, a CVS Health business. Panozzo, Marshall, and Brown are employed by Harvard Pilgrim Healthcare Institute. Aetna was reimbursed for data and analytic support from Harvard Pilgrim Healthcare Institute and the Reagan Udall Foundation for the U.S. Food and Drug Administration. Aetna receives external funding through research grants and subcontracts with Harvard Pilgrim Healthcare Institute, which are funded by the FDA, NIH, PCORI, BBCIC, Pfizer, and GSK; the Reagan-Udall Foundation for IMEDS; and PCORI for the ADAPTABLE Study.

This work was previously presented as a poster at AMCP Nexus 2018; October 22-25, 2018; in Orlando, FL.

\section{ACKNOWLEDGMENTS}

Special thanks to Sarah Malek, project manager, and Katie King, research assistant, at Harvard Pilgrim Healthcare Institute (HPHCI) for their ongoing support, and Rohan Das, data analyst at Aetna. Thanks also to the BBCIC Insulins Research Team: Berhanu Alemayehu (Merck), Bernadette Eichelberger (BBCIC), Kevin Haynes (HealthCore), Daniel J. Kent (Kaiser Permanente of Washington), Catherine M. Lockhart (BBCIC), James Marshall (HPHCI), Dorothy McCabe (Boehringer-Ingelheim), Kevin McConnell (Momenta), Cara L. McDermott (BBCIC), Cheryl N. McMahill-Walraven (Aetna), Lisa Ortendahl (HPHCI), Catherine A. Panozzo (HPHCI), Pamala A. Pawloski (HealthPartners), Brie Purcell (HPHCI), Jessica Sturtevant (HPHCI), and Jessica Young (HPHCI).

\section{REFERENCES}

1. Centers for Disease Control and Prevention. National diabetes statistics report, 2017: estimates of diabetes and its burden in the United States. 2017. Available at: https://www.cdc.gov/diabetes/pdfs/data/statistics/nationaldiabetes-statistics-report.pdf. Accessed July 21, 2019.

2. Rotenstein LS, Ran N, Shivers JP, Yarchoan M, Close KL. Opportunities and challenges for biosimilars: what's on the horizon in the global insulin market? Clin Diabetes. 2012;30(4):138-50.
3. Jacobs I, Singh E, Sewell KL, Al-Sabbagh A, Shane LG. Patient attitudes and understanding about biosimilars: an international cross-sectional survey. Patient Prefer Adherence. 2016;10:937-48.

4. Leonard E, Wascovich M, Oskouei S, Gurz P, Carpenter D. Factors affecting health care provider knowledge and acceptance of biosimilar medicines: a systematic review. J Manag Care Spec Pharm. 2019;25(1):102-12. Available at: https://www.jmcp.org/doi/10.18553/jmcp.2019.25.1.102.

5. Teeple A, Ellis LA, Huff L, et al. Physician attitudes about non-medical switching to biosimilars: results from an online physician survey in the United States. Curr Med Res Opin. 2019;35(4):611-17.

6. Teeple A, Ginsburg S, Howard L, et al. Patient attitudes about non-medical switching to biosimilars: results from an online patient survey in the United States. Curr Med Res Opin. 2019;35(4):603-09.

7. Ball R, Robb M, Anderson SA, Dal Pan G. The FDA's Sentinel initiative-a comprehensive approach to medical product surveillance. Clin Pharmacol Ther. 2016;99(3):265-28.

8. Platt R, Brown JS, Robb M, et al. The FDA Sentinel initiative-an evolving national resource. N Engl J Med. 2018;379(22):2091-93.

9. Curtis LH, Weiner MG, Boudreau DM, et al. Design considerations, architecture, and use of the Mini-Sentinel distributed data system. Pharmacoepidemiol Drug Saf. 2012;21(Suppl 1):23-31.

10. AMCP Task Force on Biosimilar Collective Intelligence Systems, Baldziki M, Brown J, et al. Utilizing data consortia to monitor safety and effectiveness of biosimilars and their innovator products. J Manag Care Spec Pharm. 2015;21(1):23-34. Available at: https://www.jmcp.org/doi/10.18553/ jmcp.2015.21.1.23.

11. Raebel M, Shetterly S, Paolino A, et al. Mini Sentinel methods. Analytic methods for using laboratory test results in active database surveillance: final report. May 16, 2016. Available at: http://www.sentinelsystem.org/ sites/default/files/Methods/Analytic_Methods_for_Using_Laboratory_Test_ Results_In_Active_Database_Surveillance_Final_Report.pdf. Accessed July 22, 2019 .

12. Gentile S, Fusco A, Colarusso S, et al. A randomized, open-label, comparative, crossover trial on preference, efficacy, and safety profiles of lispro insulin u-100 versus concentrated lispro insulin u-200 in patients with type 2 diabetes mellitus: a possible contribution to greater treatment adherence. Expert Opin Drug Saf. 2018;17(5):445-50.

13. Sorli C, Harashima SI, Tsoukas GM, et al. Efficacy and safety of onceweekly semaglutide monotherapy versus placebo in patients with type 2 diabetes (SUSTAIN 1): a double-blind, randomised, placebo-controlled, parallel-group, multinational, multicentre phase 3a trial. Lancet Diabetes Endocrinol. 2017;5(4):251-60.

14. Dalal MR, Kazemi M, Ye F, Xie L. Hypoglycemia after initiation of basal insulin in patients with type 2 diabetes in the United States: implications for treatment discontinuation and healthcare costs and utilization. Adv Ther. 2017;34(9):2083-92.

15. Dluhy RG, McMahon GT. Intensive glycemic control in the ACCORD and ADVANCE trials. N Engl J Med. 2008;358(24):2630-33.

16. Ferrannini E, Fonseca V, Zinman B, et al. Fifty-two-week efficacy and safety of vildagliptin vs. glimepiride in patients with type 2 diabetes mellitus inadequately controlled on metformin monotherapy. Diabetes Obes Metab. 2009;11(2):157-66.

17. McCall AL. Insulin therapy and hypoglycemia. Endocrinol Metab Clin North Am. 2012;41(1):57-87.

18. Rosenstock J, Marx N, Kahn SE, et al. Cardiovascular outcome trials in type 2 diabetes and the sulphonylurea controversy: rationale for the activecomparator CAROLINA trial. Diab Vasc Dis Res. 2013;10(4):289-301.

19. Gagne JJ, Glynn RJ, Avorn J, Levin R, Schneeweiss S. A combined comorbidity score predicted mortality in elderly patients better than existing scores. J Clin Epidemiol. 2011;64(7):749-59. 
20. van Walraven C, Austin PC, Jennings A, Quan H, Forster AJ. A modification of the Elixhauser comorbidity measures into a point system for hospital death using administrative data. Med Care. 2009;47(6):626-33.

21. Sun JW, Rogers JR, Her Q, et al. Adaptation and validation of the combined comorbidity score for ICD-10-CM. Med Care. 2017;55(12):1046-51.

22. Fireman B, Toh S, Butler MG, et al. A protocol for active surveillance of acute myocardial infarction in association with the use of a new antidiabetic pharmaceutical agent. Pharmacoepidemiol Drug Saf. 2012;21(Suppl 1):282-90.

23. Ginde AA, Blanc PG, Lieberman RM, Camargo CA Jr. Validation of ICD-9-CM coding algorithm for improved identification of hypoglycemia visits. BMC Endocr Disord. 2008;8:4.

24. Ghouse J, Skov MW, Kanters JK, et al. Visit-to-visit variability of hemoglobin Alc in people without diabetes and risk of major adverse cardiovascular events and all-cause mortality. Diabetes Care. 2019;42(1):134-41.

25. Young JB, Gauthier-Loiselle M, Bailey RA, et al. Development of predictive risk models for major adverse cardiovascular events among patients with type 2 diabetes mellitus using health insurance claims data. Cardiovasc Diabetol. 2018;17(1):118.

26. Cournot M, Burillo E, Saulnier PJ, et al. Circulating concentrations of redox biomarkers do not improve the prediction of adverse cardiovascular events in patients with type 2 diabetes mellitus. J Am Heart Assoc. 2018;7(5).

27. Currie CJ, Poole CD, Evans M, Peters JR, Morgan CL. Mortality and other important diabetes-related outcomes with insulin vs other antihyperglycemic therapies in type 2 diabetes. J Clin Endocrinol Metab. 2013;98(2):668-77.

28. Holman RR, Paul SK, Bethel MA, Matthews DR, Neil HA. 10-year follow-up of intensive glucose control in type 2 diabetes. N Engl J Med. 2008;359(15):1577-89.

29. Perez-Nieves M, Kabul S, Desai U, et al. Basal insulin persistence, associated factors, and outcomes after treatment initiation among people with type 2 diabetes mellitus in the U.S. Curr Med Res Opin. 2016;32(4):669-80.

30. Perez-Nieves M, Boye KS, Kiljanski J, Cao D, Lage MJ. Adherence to basal insulin therapy among people with type 2 diabetes: a retrospective cohort study of costs and patient outcomes. Diabetes Ther. 2018;9(3):1099-11.

31. Intensive blood-glucose control with sulphonylureas or insulin compared with conventional treatment and risk of complications in patients with type 2 diabetes (UKPDS 33). UK Prospective Diabetes Study (UKPDS) Group. Lancet. 1998;352(9131):837-53.

32. Bonafede M, Chandran A, DiMario S, Saltiel-Berzin R, Saliu D. Medication usage, treatment intensification, and medical cost in patients with type 2 diabetes: a retrospective database study. BMJ Open Diabetes Res Care. 2016;4(1):e000189.

33. Cramer JA. A systematic review of adherence with medications for diabetes. Diabetes Care. 2004;27(5):1218-24.
34. Kollhorst B, Behr S, Enders D, Dippel FW, Theobald K, Garbe E. Comparison of basal insulin therapies with regard to the risk of acute myocardial infarction in patients with type 2 diabetes: an observational cohort study. Diabetes Obes Metab. 2015;17(12):1158-65.

35. Duckworth W, Abraira C, Moritz T, et al. Glucose control and vascular complications in veterans with type 2 diabetes. N Engl J Med. 2009;360(2):129-39.

36. Roumie CL, Greevy RA, Grijalva CG, et al. Association between intensification of metformin treatment with insulin vs sulfonylureas and cardiovascular events and all-cause mortality among patients with diabetes. JAMA. 2014;311(22):2288-96.

37. Green JB, Bethel MA, Armstrong PW, et al. Effect of sitagliptin on cardiovascular outcomes in type 2 diabetes. N Engl J Med. 2015;373(3):232-42.

38. Action to Control Cardiovascular Risk in Diabetes Study Group, Gerstein HC, Miller ME, et al. Effects of intensive glucose lowering in type 2 diabetes. N Engl J Med. 2008;358(24):2545-59.

39. Marso SP, Daniels GH, Brown-Frandsen K, et al. Liraglutide and cardiovascular outcomes in type 2 diabetes. N Engl J Med. 2016;375(4):311-22.

40. ORIGIN Trial Investigators, Gerstein HC, Bosch J, et al. Basal insulin and cardiovascular and other outcomes in dysglycemia. N Engl J Med. 2012;367(4):319-28.

41. Lipska KJ, Parker MM, Moffet HH, Huang ES, Karter AJ. Association of initiation of basal insulin analogs vs neutral protamine Hagedorn insulin with hypoglycemia-related emergency department visits or hospital admissions and with glycemic control in patients with type 2 diabetes. JAMA. 2018;320(1):53-62.

42. Solomon MD, Vijan S, Forma FM, Conrad RM, Summers NT, Lakdawalla DN. The impact of insulin type on severe hypoglycaemia events requiring inpatient and emergency department care in patients with type 2 diabetes. Diabetes Res Clin Pract. 2013;102(3):175-82.

43. Simeone JC, Nordstrom BL, Appenteng K, Huse S, D'Silva M. Replication of Mini-Sentinel study assessing mirabegron and cardiovascular risk in nonMini-Sentinel databases. Drugs Real World Outcomes. 2018;5(1):25-34.

44. Lissenden B. The effect of cancer diagnosis on switching health insurance in Medicare. Health Econ. 2018;28(3):339-49.

45. Rahman M, Keohane L, Trivedi AN, Mor V. High-cost patients had substantial rates of leaving Medicare Advantage and joining traditional Medicare. Health Aff (Millwood). 2015;34(10):1675-81.

46. Lewis JD, Bilker WB, Weinstein RB, Strom BL. The relationship between time since registration and measured incidence rates in the General Practice Research Database. Pharmacoepidemiol Drug Saf. 2005;14(7):443-51.

47. Mamtani R, Haynes K, Finkelman BS, Scott FI, Lewis JD. Distinguishing incident and prevalent diabetes in an electronic medical records database. Pharmacoepidemiol Drug Saf. 2014;23(2):111-18. 